\title{
Completion of the FK5 Extension and a preliminary investigation of the FK5 system
}

\author{
H. Schwan \\ Astronomisches Rechen-Institut \\ Mönchhofstraße 12-14 \\ D-6900 Heidelberg 1 \\ Germany
}

\begin{abstract}
The construction of the Fifth Fundamental Catalogue (FK5) is shortly described with emphasis on the system of the Basic FK5 and on the completion of the FK5 Extension. New observations have revealed systematic errors mainly in the declination system of the FK5 and in the right ascensions south of $-50^{\circ}$. Comparisons of the new observations with the Basic FK5 and the FK5 Extension (made separately for the bright and faint stars) have shown that the FK5 Extension is essentially on the system of the Basic FK5.
\end{abstract}

\section{Introduction}

The construction of the new fundamental system was initiated by Fricke (1974) soon after new observations had revealed considerable systematic errors in the FK4, in particular for the southern right ascensions (Anguita, 1974). Since about 150 catalogues of observations not yet included in the FK4 (Fricke et al., 1963) had appeared after 1950, Fricke stressed also the necessity to improve the individual precision of each fundamental star and, in addition, the extension of the fundamental system to fainter magnitudes. At the XV General Assembly held in Sydney, 1973, the International Astronomical Union has recommended that the improvement of the FK4 and its extension to a fainter magnitude limit shall be carried out at the Astronomisches Rechen-Institut, Heidelberg.

Work started with the collection of new observations and the identification of all observations with the FK4 catalogue, the FK4 Supplement (Fricke, 1963), the AGK3R (Smith, 1980) and SRS (Smith et al., 1990), which served as so-called master catalogues for the documentation of the relevant observations. In addition, new analytical methods have been developed for analyzing these data and for deriving the systematic corrections to the FK4 system (Brosche (1966), Schwan (1977), Bien et al. (1978), Schwan (1983)). Progress reports on the work on the FK5 have been given continuously, e.g. by Fricke (1980a, 1985), Fricke and Gliese (1978), Schwan (1988, 1990, 1991) and Corbin and Schwan (1990).

After Corbin had already determined proper motions for all AGK3R stars agreement was achieved with the US Naval Observatory that work on the faint stars to 
be included in the FK5 Extension should be made at US Naval Observatory using also Corbin's results for the AGK3R. Mean positions and and proper motions for the FK4 Supplement stars were derived at Astronomisches Rechen-Institut.

The Basic FK5, giving improved positions and proper motions for the classical 1535 fundamental stars, was published by Fricke et al. (1988). With the publication of the printed version of the FK5 Extension (Fricke et al., 1991) work on the whole FK5 is completed.

In view of the new concepts developed in the past few years for establishing a new primary reference frame we want to start with a short discussion on the basic properties of the FK5 system. A few remarks concerning the estimated precision of positions and proper motions in the FK5 are added, and a short description of the construction of the FK5 Extension and its main characteristics are given. Finally a preliminary investigation of the systems of the Basic FK5 and of the FK5 Extension is presented, based on modern observations not yet included in the FK5.

\section{Basic principles for constructing the system of the FK5}

The establishment of a reference coordinate system is one of the primary tasks of astrometry. With the VLBI techniques and their ability to measure angular distances on the mas-level new concepts have been developed for constructing a reference system from VLBI observations of quasi-stellar extragalactic radio sources. At the 127th IAU Colloquium held in Virginia Beach (1990) it was recommended to adopt an extragalactic system as the primary new conventional celestial reference frame. It will be represented by the radio positions of a selected number of primary radio sources (Recommendation VI). The direction to these objects are postulated to define (within the precision of the VLBI measurements) fixed points on the sphere since the objects are assumed to be at cosmological distances. The radio system is therefore purely kinematically defined without any sophisticated theory for the motion of the objects representing the system.

Since the radio reference frame is at present not well accessable in other than radio wavelengths and since it is represented by a comparatively small number of objects, there are other systems in practical use. One of them is the "Solar System frame" which is represented by the ephemerides of objects of the Solar System. These ephemerides were constructed by adopting a dynamical theory for the motion of the objects and fitting the constants to the observations. The Solar System frame is therefore a dynamically defined reference systern.

In optical wavelengths the FK5 catalogue is still representing the celestial reference frame as long as its relationship to the extragalactic frame is not sufficiently accurate determined (see Note 5 for Recommendation VII in the Proceedings of the 127th IAU Coll., p. 417). In the following we want shortly to describe the principles on which the FK5 system is based in order to show that it cannct be characterized as a purely kinematical or dynamical system. In this context reference has to be made to the much more extensive discussions by Fricke $(1972,1975,1981)$.

The system of the FK5 has been derived by adoping the FK4 as a provisional system and determining systematic corrections to that system. The corrections concern the equinox and equator of the FK4 and their fictitious motions, the elim- 
ination of regional errors in the mean FK4 positions and proper motions, and the introduction of new constants, in particular the constant of luni-solar precession.

\subsection{CORRECTION OF THE FK4 EQUINOX AND EQUATOR}

In a series of papers Fricke $(1979,1980 \mathrm{a}, 1980 \mathrm{~b}, 1981,1982)$ has reported on his work for improving the FK4 equinox and equator. 'The possible errors of the FK4 equator were studied by an examination of fundarnental observations, including also observations of the Sun and planets. The determination of the FK5 equator on the basis of observations of objects of the planetary system is essentially a dynamical determination, since the relevant observations and their interpretation are combined with the dynamics of the planetary system. No significant correction could be determined from the available observations and the FK4 equator remained therefore unchanged.

A remark concerning the importance of the FK5 for the future Hipparcos catalogue may be in order. Since the Hipparcos satellite measures only angular differences between stars the zero points in position and proper motion are arbitrary. One possibility to fix the zero points is to rotate the Hipparcos sphere on the FK5. This process defines the celestial pole on the Hipparcos sphere and the equinox in the associated equatorial plane. Although the Hipparcos measurements cannot define these quantities themselves they can, on the other hand, determine all of the systematic errors in the FK5 which are not represented by a rigid rotation. In particular a shift of the FK5 equator could thus be detected.

For the determination of the equinox correction Fricke has adopted three different approaches. The first one uses the same techniques as for the determination of the equator. Discussing 35 individual equinox determinations made in the present century from observations of the Sun, planets and minor planets, Fricke (1982) has found that the FK4 equinox needs a correction of +0.042 at the epoch 1958.5 (this correction includes only the observations from 1950 onwards) and that the FK4 equinox rotates by $0.085 / c y$ with respect to the dynamical equinox.

Another method uses the timings of lunar occultations of stars. From these observations one can derive corrections to the adopted stellar system (namely the FK4, if the star positions are reduced to that system) and to the adopted lunar ephemeris. Occultations of the past about 150 years have indicated a correction of 0.052 to the FK4 equinox at 1960 and a fictitious motion of $0.087 / c y$ with respect to the dynamical equinox has been found.

The third method is a kinematical method which uses the FK4 proper motions themselves. The FK4 proper motions allow one to determine three components of angular velocity around the coordinate axes. Two of these rotations are interpreted as galactic rotation and as resulting from the use of an erroneous precessional constant in the reduction of the observations. The third component, a rotation around the z-axis, has been assumed to arise from a motion of the FK4 equinox relative to the dynamical equinox. Fricke derived the value $0.082 / c y$ for this motion. It is remarkable how well the results obtained by the different methods agree with each other.

The mean value $\dot{E}=0.085 / c y$ was adopted for the correction of the motion of 
the FK4 equinox so that the full correction $\mathrm{E}(\mathrm{T})$ reads

$E(T)=0.035+0.085 / c y(T-19.50)$.

The value $\mathrm{E}$ at the mean epoch 1958.5 results from dynamical determinations alone whereas the correction for the motion of the FK4 equinox (and therefore $\mathrm{E}(\mathrm{T})$ ) includes also kinematical determinations.

\subsection{IMPROVEMENT OF THE CONSTANT OF LUNI-SOLAR PRECESSION}

The use of a wrong precessional constant in the reduction of the observed star positions to a common equinox indroduces corresponding systernatic errors in the derived proper motions which will consequently rotate with respect to an inertial system. Since this error can easily be modelled as a function of the position on the sphere one can determine the correction to the precessional constant used in a fundamental catalogue by adjusting its proper motions to the model. In the FK5 the correction +1 . $10 / c y$ to Newcomb's value of luni-solar precession was adopted as determined by Fricke (1977) from an analysis of the FK4 and N30 proper motions. This correction to the FK4 proper motion system is essentially kinematically determined. The crucial point in the determination is the model adopted for the motions of the stars under consideration. Deviations of their real motion from the adopted model will severely bias the values obtained for the constants to be determined. Mainly from LLR observations (J.G.Williams et al. (1991)) there is strong evidence that Fricke's correction is too large by nearly $0.3 / c y$. The conceptional superiority of LLR techniques (and VLBI after a sufficiently long period of observations) for determining the precessional constant is that the motion of the observed objects are well known. In the context of determining precession and galactic rotation reference is also made to the paper by Miyamoto et al. (this proceedings)

A small correction to the planetary precession was dynamically determined from improved planetary masses. The expressions for the constant of general precession depend therefore both on kinematical and dynamical determinations.

\subsection{REGIONAL CORRECTIONS TO THE FK4 SYSTEM}

From about 85 absolute and quasi-absolute catalogues observed in the present century systematic corrections to the FK4 positions and proper motions were determined as a function of the right ascension and declination (and the apparent magnitude in the case of the $\alpha$-system). In a first step each catalogue was reduced to the equinox and equator of the FK4 by subtracting the mean equatorial deviation $(\Delta \alpha)_{0}$ (Cat-FK4) from the catalogue right ascensions, and by eliminating the mean deviation $(\Delta \delta)_{0}$ from the declinations according to the formula given by Morgan (1952). The differences Cat-FK4, thus reduced to the FK4 equinox and equator, were used to determine and eliminate local distortions in the FK4 system in order to get a more rigid sphere which was then rotated on the FK5 equinox and equator. The regional corrections are given in the appendix to the FK5 (Part I, p. 85ff).

In summary the FK5 system has been constructed by applying, dynamical as well as kinematical concepts. It is a hybrid system and cannot be classified as belonging to only one type of reference systems. 


\section{The improvement of the individual accuracy of each FK4 star}

The residuals $\Delta \alpha, \Delta \delta$ (Cat-FK4) after elimination of the systematic differences contain information on the accidental error in the FK4 position of a particular star at the epoch of the new observation. This observation can be used to improve the FK4 position and proper motion without re-discussing the old observations (already included in the FK4) in a way which is theoretically strictly equivalent to a least squares solution using the old and new data individually (see e.g. Kopff et al., 1964). This procedure was already described in the GC by Boss (1937) and it had been adopted in the construction of the FK4. We have again applied the same procedure for the individual improvement of the precision in mean position and proper motion of each FK4 star. From a practical point of view and since, moreover, this procedure has been applied twice, one may question whether the process works really successfully. If we look at the mean epochs in the FK4 and FK5, respectively, then we find a large shift of nearly 40 years in right ascension and nearly 30 years in declination. Possible reasons for this shift. are:

a) Comparatively many new observations have become available, in particular in right ascension.

b) The modern observations are (much) more precise than the older ones and received therefore comparatively high weight in the least squares solutions.

c) The new observations are not completely independent from the FK4. This may be a consequence of using the FK4 itself in the reduction of the observations.

d) The estimated mean errors in the FK4, which had to be used for including the new observations, are not correct.

e) The weighting system applied for the new observations was not fully consistent with the old one.

f) The error law adopted for determining weights, narnely

$\epsilon_{n}=\epsilon_{0}\left(a^{2}+b^{2} / n\right)^{-1 / 2}$

is not strictly valid in practical applications. For the determination of weights and the derivation of individual corrections reference is also made to Bien (1987), Kopff et al. (1964) and Wahl (1937).

Some of the given arguments can explain the large shift of mean epochs as real, but there are other arguments showing the problems involved in the procedure of just incorporating new observations into a mean catalogue position and proper motion. Although no final results of the Hipparcos mission are at present available there is some indication that the error estimates in the Basic FK5 may be too optimistic (see Walter, this proceedings).

\section{The construction of the FK5 Extension}

The construction of the FK5 Extension had been initiated by Fricke (1974) mainly to extend the fundamental system to fainter magnitudes and also to increase the star density. It was decided to select the new fundamental stars from the IRS list (AGK3R and SRS stars) for the faint part of the FK5 Extension. The intermediate gap in the magnitude distribution between the bright stars of the Basic FK5 and the faint stars in the FK5 Extension was filled with stars form the FK4 Supplement 
(Fricke, 1963). Since Corbin (1977) had already determined proper motions for all the AGK3R stars agreement was achieved between ARI and USNO that the faint stars to be included into the FK5 Extension should be selected from Corbin's work of determining mean positions and proper motions for all IRS stars. At ARI mean positions and proper motions for the FK4 Supplement stars were derived.

A lot of catalogues giving star positions of the past 100 years were identified with the stars in the FK4, FK4 Supplement and IRS in order to find their histories of observation. All relevant observations were referred to the equinox and equator for B1950.0 still adopting Newcomb's precessional quantities, and they were then transformed to the FK4 system, which was adopted as a preliminary system. Since the FK4 Supplement stars are essentially within or near the magnitude limit of the FK4, these stars could be transformed directly to the FK4 system by making use of the FK4 observations in a catalogue.

For transforming the IRS stars with their magnitudes from about 6.5 to 9.5 it was, however, first necessary to construct so-called Base Systems which represent the FK4 system at fainter magnitudes. These Base Systems could then be used instead of the FK4 itself to determine the systematic relation between a catalogue of observation and the FK4. Since the work on the IRS proper motions and the construction of the Base Systems is described by Corbin (1978) and Corbin and Urban (1990) a comparatively brief description may be sufficient here.

The Base System north of $-30^{\circ}$ (NBAS) was constructed from 10 catalogues observed with screens. These observations were assumed to be free of magnitude equation and mean positions and proper motions were derived for 5590 stars representing the FK4 system north of $-30^{\circ}$. South of $-30^{\circ}$ only three catalogues observed with screens were available, an insufficient number to derive proper motions. This problem could be solved since it had been found from work on the northern hemisphere that observations made with moving wires have no magnitude equation depending on the declination. The faint southern stars in such a catalogue could therefore be reduced to the FK4 system by determining the magnitude equation north of $-30^{\circ}$ (using the NBAS) and extrapolating this magnitude equation to more southern declinations. Seven additional catalogues were in that way reduced to the FK4 system and a Southern Base System (SBAS) of mean positions and proper motions for 4708 stars was derived. With the NBAS, SBAS, the FK4 itself and the improved FK4 Supplement (which had already become available at Astronomisches Rechen-Institut) other catalogues, not observed with screens or moving wires, were transformed to the FK4 system. Altogether there were finally about 200 catalogues available for inclusion in the FK5 Extension.

Weighted least squares solutions were performed and the new fundamental stars were selected according to their distribution on the sky and with respect to apparent magnitude, and also taking into account the estimated mean errors of positions and proper motions. There are 3117 stars included in the FK5 Extension, 992 stars from the FK4 Supplement and 2125 from the IRS.

The mean positions and proper motions, which were primarily obtained in the system of the FK4 and still based on Newcomb's precessional values were then transformed to the IAU(1976) System of Astronomical Constants, and the systematic corrections FK5-FK4 (see FK5, Part I, p. 85ff) were applied. 
TABLE I

Average Precision of the FK5 Extension

$\begin{array}{llc} & \text { R.A. } & \text { Decl. } \\ \text { Epoch } & 1946.2 & 1941.9 \\ \epsilon(\text { pos }) & 0.0034 & 0.059 \\ \epsilon(\text { prop.mot. }) & 0.0162 / c y & 0.267 / c y\end{array}$

The FK5 Extension is a rather inhomogeneous catalogue with respect to the distribution of mean epochs and mean errors of mean positions and proper motions because the stars in the IRS and the FK4 Supplement have significantly different histories of observation. Moreover the stars north of about $-30^{\circ}$ are on the average better observed than those in more southern declinations. In Table I mean values of these quantities averaged over all Extension stars are given; more detailed distributions can be found in Corbin and Schwan (1991).

With the completion of the FK5 Extension (Fricke et al., 1991) the whole FK5 (Basic + Extension) contains 4652 stars providing a reference system with about 3 stars per 25 square degrees up to apparent magnitude 9.5 .

\section{A preliminary investigation of the FK5 system}

New observations not yet included in the FK5 have become available allowing one to get information on the FK5 system. The present investigation uses observations published in the Carlsberg Meridian Catalogues LP4, LP5, LP6 observed at La Palma (CMC 1989, 1991, 1992), in the Tokyo PMC Catalogues for 1985-1988 (Yoshizawa et. al. (1987); Yoshizawa and Suzuki (1989, 1991); Yoshizawa et. al. (1992)), in Perth 83 (Harwood, 1990), in addition to results obtained with the meridian circles at Bordeaux around 1986 (Réquième, 1988), at Santiago from 1979 to 1989 (Carrasco and Loyola, 1990), with the 7-inch meridian circle of the US Naval Observatory operated at present at Black Birch, New Zealand (see IAU Transactions), and with the astrolabe at Santiago in 1976 (Noël and Débarbart, 1990).

\subsection{POSSIBLE SYSTEMATIC ERRORS IN THE BASIC FK5}

First evidence on systematic errors in the Basic FK5 was reported by Morrison et al. (1991). Observations made subsequently and also with different instruments confirmed the negative defect in the declination system of the F K5 from $+40^{\circ}$ to $+60^{\circ}$ with a maximum deviation up to -0.1 for some catalogues.

The deviations of modern observations from the FK5 as a function of the declination are shown in Fig. 1 for the right ascensions, and in Fig. 2 for the declinations. There are wavelike features $\Delta \alpha_{\delta} \cos \delta$ north of $-30^{\circ}$ with an amplitude up to about \pm 0 s.003. The average deviation of these observations at their mean epoch (about 1987 ) is, however, in general smaller than \pm 0 s.002, except for the region north of $+75^{\circ}$ where the amplitude exceeds $+0^{s} 005$. South of $-30^{\circ}$ Perth 83 and the Santiago meridian circle observations show a positive trend from $-50^{\circ}$ to the southern 
pole with an amplitude of at least +0.010 . This trend has been confirmed by the 7 -inch TC-observations made by the US Naval Observatory at Black Birch.

$\Delta \alpha_{\delta} \cos \delta[0.001]$

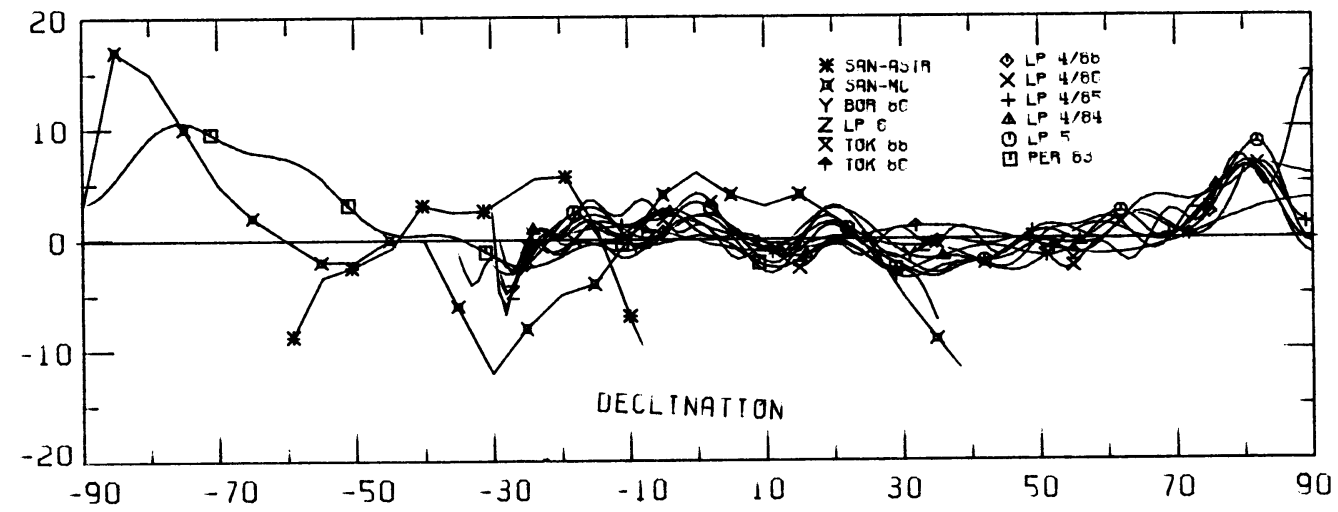

Fig. 1. Systematic differences $\Delta \alpha \cos \delta$ as a function of the declination between the Basic FK5 and modern observations; Units: [0s.001]

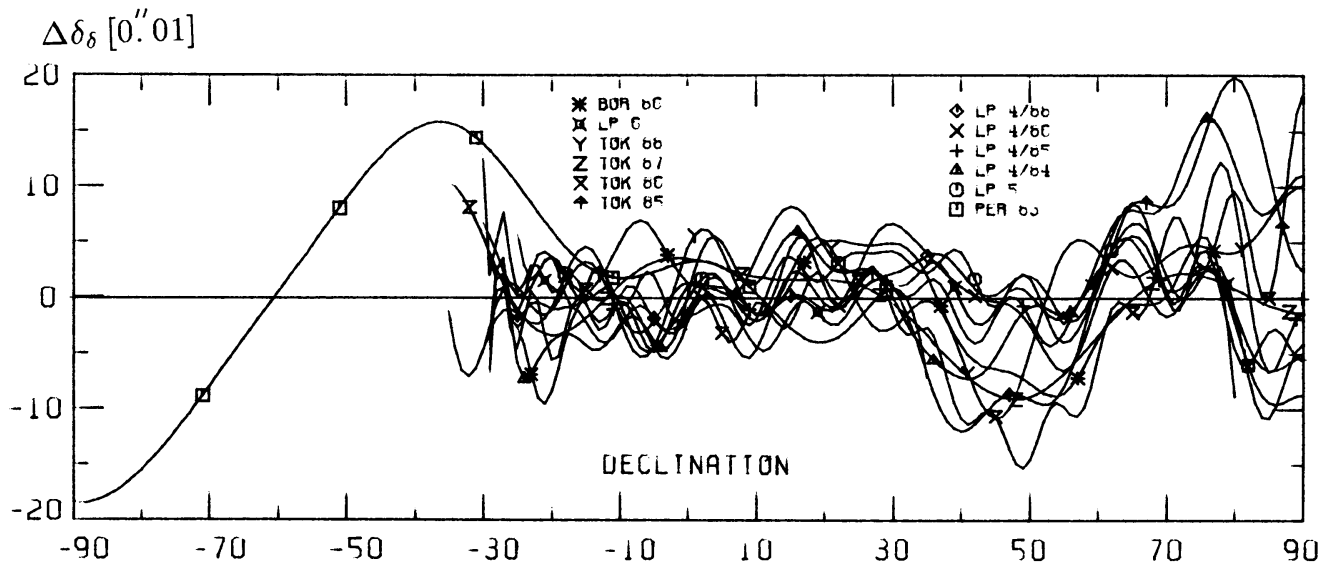

Fig. 2. Systematic differences $\Delta \delta$ as a function of the declination between the Basic FK5 and modern observations; Units: [0."01]

In declination the dispersion between the various catalogues is larger than in right ascension. From $-30^{\circ}$ to $+40^{\circ}$ we have indication for wave-like errors in the FK5 with a mean deviation not larger than \pm 0.03 at the epoch 1987 . The most significant deviation is from $+40^{\circ}$ to $+60^{\circ}$ with a mean value exceeding -0.05 . North of $+60^{\circ}$ there is a positive trend up to about +0.06 near $+80^{\circ}$. In the southern sky Perth 83 and the preliminary results obtained from the Black Birch expedition show a positive feature in the zone $-30^{\circ}$ to $-55^{\circ}$. The large negative deviation south of $-60^{\circ}$ may be a peculiarity in Perth 83 . 


\subsection{THE SYSTEM OF THE FK5 EXTENSION}

As described in Section 4 it was necessary to make certain assumptions on the magnitude equation in the catalogues included in the Base System. Comparisons of the FK5 Extension with modern observations (which can be assumed to be free of magnitude dependent systematic errors) give information on whether or not the new fundamental stars in the FK5 Extension are on the system of the Basic FK5. In addition we can check whether there is a magnitude equation in the FK5 Extension by comparing its bright and faint stars separately with the new observations.

Not all of the catalogues included in Figs. 1 and 2 for the examination of the system of the Basic FK5 could also be used to investigate the system of the FK5 Extension. The reason is mainly that many of those catalogues have no or not enough Extension stars for determining their systematic relation to the FK5 Extension. Moreover the number of observations per Extension star is in general much smaller than for the stars in the Basic FK5; the dispersion of the residuals is therefore larger and the system not so well defined. In the Figs. 3 through 6 one finds those comparisons which could be made with some confidence.

Fig. 3 (upper part) shows the systematic differences in right ascension between LP4 and the FK5 separateley for the Basic FK5 (LP4 observations made in 1984) and the bright and faint stars in the FK5 Extension, respectively. Except for the faint stars near $+75^{\circ}$ all comparisons agree very well with each other and the right ascension system of the FK5 Extension should agree therefore with that of the Basic FK5. The comparison Tokyo PMC 86 - FK5 (Fig. 3, lower part) confirms that the system of the bright stars in the FK5 Extension agrees with the system of the Basic FK5; the discrepancy north of $+70^{\circ}$ may be a systematic error in Tokyo PMC 86. There are only about 80 faint Extension stars observed in this catalogue and no systematic relation could therefore be determined for fainter magnitudes. In the southern sky Perth 83 indicates no systematic deviation between the Basic FK5 and the bright and faint stars in the FK5 Extension (Fig. 5). South of $-40^{\circ}$ too few faint Extension stars were observed in Perth 83 in order to permit a catalogue comparison.

It had already been found from the comparisons of the new observations with the Basic FK5 (see Figs. 1 and 2) that the dispersion between the various declination systems is large compared with the right ascensions. This holds even more for the systematic differences with respect to the FK5 Extension because of the much smaller number of observed stars and the smaller number of observations per star. The conclusions on the declination system of the FK5 Extension are, therefore, not so clear as for the right ascenisons.

The comparison in declination between LP4 and the FK5 is given in Fig. 4 (upper part) separately for the Basic FK5 (LP4 observations made in 1984) and for the bright and faint stars in the FK5 Extension, respectively. The agreement between all three curves is satisfactory, except for the faint stars near the northern pole and the bright stars near $-10^{\circ}$. In the case of Tokyo PMC, 86 (Fig. 4, lower part) the comparison with the bright stars could only be used for $\delta>-20^{\circ}$ since for the more southern declinations the dispersion of residuals increased considerably and the Tokyo system became unrealistic. Tokyo PMC 86 confirms that the bright 
stars in the FK5 Extension are on the system of the Basic FK5. As for the right ascensions no systematic relation could be determined for the fainter magnitudes.
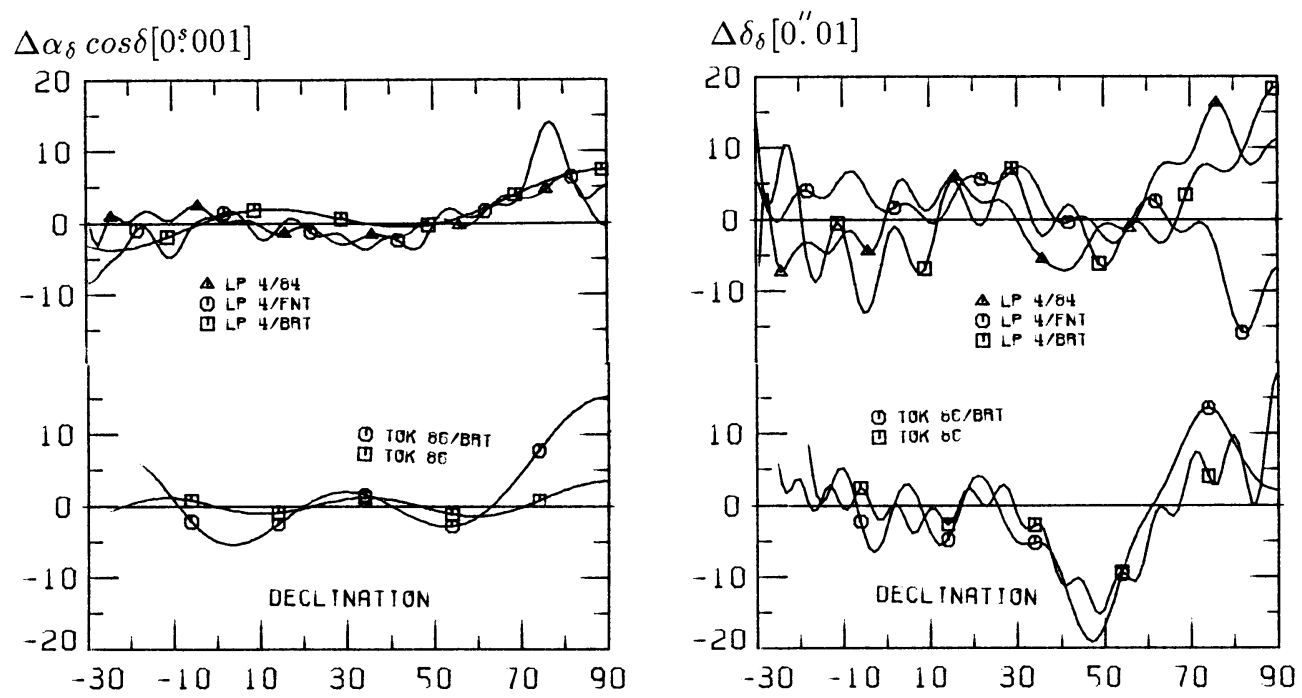

Fig. 3 (left) and Fig. 4(right). Systematic differences $\Delta \alpha_{\delta} \cos \delta$ (Fig. 3) and $\Delta \delta_{\delta}$ (Fig. 4) between the FK5 and modern observations separately for the Basic FK5 and the bright and faint stars in the FK5 Extension. Upper part: Carlsberg LP4, lower part: Tokyo PMC 86. Units: [0. 001$]$ and $[0.01]$ in $\alpha$ and $\delta$, respectively
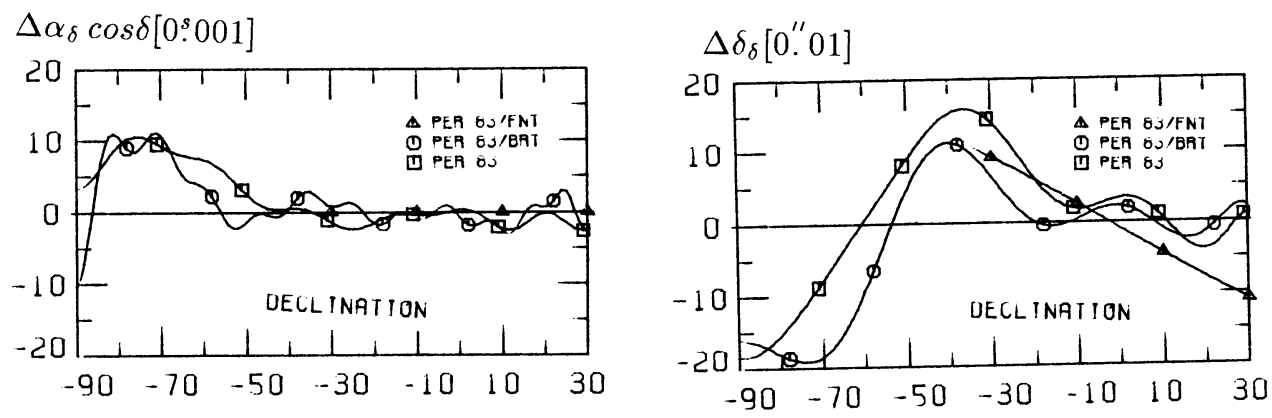

Fig. 5 (left) and Fig. 6 (right). Systematic differences $\Delta \alpha_{\delta} \cos \delta$ (Fig. 5 ) and $\Delta \delta_{\delta}$ (Fig. 6 ) between the FK5 and Perth 83 separately for the Basic FK5 and the bright and faint stars in the FK5 Extension. Units: [0.001] and [0."01] in $\alpha$ and $\delta$, respectively

The comparison of Perth 83 with the FK5 (Fig. 6) indicates no significant systematic deviation between the Basic FK5 and FK5 Extension. As for the right ascensions no systematic relation could be determined for the faint Extension stars south of $-40^{\circ}$. 


\section{Conclusions}

Comparisons of the Basic FK5 with modern observations not yet included in the FK5 have revealed systematic errors of the FK5 in some parts of the sky. In particular the declination system shows significant errors north of about $+40^{\circ}$ and in the southern sky from $-30^{\circ}$ to $-50^{\circ}$. The largest systematic deviation in right ascension is south of $-50^{\circ}$ with an amplitude of about +0.010 . With very few exceptions no systematic differences between the systems of the Basic FK5 and the FK5 Extension could be detected from the new observations.

\section{References}

Anguita, C. (1974), in IAU Symp. No. 61, New problems in Astrometry, held in Perth, Western Australia, 13-17 August, 1973, eds. W. Gliese, C.A. Murray, R.H. Tucker, p. 63

Bien, R., Fricke, W., Lederle, T., Schwan, H. (1978), Veröff. des Astron. Rechen-Instituts, Heidelberg, Nr. 29, Verlag G. Braun, Karlsruhe

Bien, R. (1987), Astron. Astrophys., Vol. 188, p. 225

Boss, B. (1937), General Catalogue of 33,342 Stars for the Epoch 1950, Carnegie Institution, Washington, Vol. I, p. $49 \mathrm{ff}$

Brosche, P. (1966), Veröff. des Astron. Rechen-Instituts, Heidelberg, Nr. 17, Verlag G. Braun, Karlsruhe

Carrasco, G., Loyola, P. (1990), in IAU Symp. No. 141, Inertial Coordinate System on the Sky, held in Leningrad, USSR, 17-21 October 1989, eds. J.H. Lieske, V.A. Abalakin, p. 385

CMC 1989, 1991, 1992, Carlsberg Meridian Catalogue La Palma, Nos. 4, 5, 6; Copenhagen University, Royal Greenwich Observatory, Real Instituto y Observatorio de la Armada en San Fernando

Corbin, T. (1977), The Proper Motion System of the AGK3R, A dissertation presented at the Graduate Faculty of the Univerity of Virginia in Candidacy for the Degree of Doctor of Philosophy, Leander McCormick Observatory, University of Virginia

Corbin, T. (1978), in IAU Coll. No. 48, Modern Astrometry held in Vienna, Austria, 11-14 September 1978, eds. F.V. Prochazka, R.H. Tucker, p. 505

Corbin, T., Urban, S. (1990), in IAU Symp. No. 141, Inertial Coordinate System on the Sky, held in Leningrad, USSR, 17-21 October 1989, eds. J.H. Lieske, V.A. Abalakin, p. 433

Corbin, T., Schwan, H. (1991), in IAU Coll. No. 127, Reference Systems, held in Virginia Beach, VA, USA, 14-20 October 1990, eds. J.A. Hughes, C.A. Smith, G.H. Kaplan, p. 238

Fricke, W., Kopff, A. (1963), Veröff. des Astron. Rechen-Instituts, Heidelberg, Nr. 10, Verlag G. Braun, Karlsruhe

Fricke, W. (1963), Veröff. des Astron. Rechen-Instituts, Heidelberg, Nr. 11, Verlag G. Braun, Karlsruhe

Fricke, W. (1972), Annual Rev. of Astronomy and Astrophys., Vol. 10, p. 101

Fricke, W. (1974), in IAU Symp. No. 61, New problems in Astronetry, held in Perth, Western Australia, 13-17 August, 1973, eds. W. Gliese, C.A. Murray, R.H. Tucker, p. 23

Fricke, W. (1975), in IAU Coll. No. 26, On Reference Coordinate Systems for Earth Dynamics, eds. B. Kolaczek, G. Weiffenbach, p. 201,

Fricke, W. (1977), Veröff. des Astron. Rechen-Instituts, Heidelberg, No. 28, Verlag G. Braun, Karlsruhe

Fricke, W., Gliese, W. (1978), in IAU Coll. No. 48, Modern Astrometry held in Vienna, Austria, 11-14 September 1978, eds. F.V. Prochazka, R.H. Tucker, p. 421

Fricke, W. (1979), in IAU Symp. No. 81, Dynamics of the Solar System, held in Tokyo, Japan, 23-26 May 1978, ed. R.L. Duncombe, p.133

Fricke, W. (1980a), in Mitteilungen der Astron. Gesellschaft, No. 48, Astrometrie und Dynamische Astronomie, Hamburg, p. 29

Fricke, W. (1980b), Celestial Mechanics, Vol. 22, 113

Fricke, W. (1981), in IAU Coll. No. 56, Reference Coordinate Systems for Farth Dynamics, held in Warsau, Poland, 8-12 Sept. 1980, eds. E.M. Gaposchkin, B. Kolaczek, p.

Fricke, W. (1982), Astron. Astrophys., Vol. 107, L13 
Fricke, W. (1985), Celest. Mech., Vol. 36, 207

Fricke, W., Schwan, H., Lederle, T., in collaboration with Bastian, U., Bien, R., Burkhardt, G., du Mont, B., Hering, R., Jährling, R., Jahreiß, H., Röser, S., Schwerdtfeger, H.M., Walter, H.G. (1988), Veröff. des Astron. Rechen-Instituts, Heidelberg, No. 32, Verlag G. Braun, Karlsruhe

Fricke, W., Schwan, H., Corbin, T., in collaboration with Bastian, U., Bien, R., Cole, C., Jackson, E., Jährling, R., Jahreiß, H., Lederle, T., Röser, S., 1991, Veröff. des Astron. Rechen-Instituts, Heidelberg, No. 33, Verlag G. Braun, Karlsruhe

Harwood, D.N. (1990), Perth 83, a Catalogue of Positions of 12,263 Stars, derived from observations during the years 1980 through 1987 at the Perth Observatory, Bickley, W.A.; in collaboration with C.Bowers, S. Ewing, M. Sultana, A. Verveer; on magnetic tape

IAU Transactions, Vol. 21, Commission 8 (in press)

Kopff, A., Nowacki, H., Strobel, W. (1964), Veröff. des Astron. Rechen-Instituts, Heidelberg, Nr. 14, Verlag G. Braun, Karlsruhe

Morgan, H.R. (1952), Catalogue of 5,268 Standard Stars, 1950.0, Based on the Normal System N30, Astron. Papers, Vol. XIII, Part II, Washington, p. XI

Morrisson, L.V., Gibbs, P., Helmer, L., Fabricius, C., Einicke, O., Réquième, Y., Rapaport, M. (1991), in IAU Coll. No. 100, Fundamentals of Astrometry, held in Belgrade, Yugoslavia, 8-11 September 1987, eds. H.K. Eichhorn, C.A. Murray, Astrophys. Space Sci. No. 177, p. 31

Noël, F., Débarbart, S. (1990) Astron. Astrophys., Vol. 232, 267

Réquième, Y. (1988) catalogue received on magnetic tape (private communication)

Schwan, H. (1977), Veröff. des Astron. Rechen-Instituts, Heidelberg, No. 29, Verlag G. Braun, Karlsruhe

Schwan, H. (1983), Veröff. des Astron. Rechen-Instituts, Heidelberg, No. 30, Verlag G. Braun, Karlsruhe

Schwan, H. (1988), in IAU Symp. No. 133, Mapping the Sky, held in Paris, France, 1-5 June 1987, eds. S. Débarbart, K.H. Eichhorn, A.R. Upgren, p. 151

Schwan, H. (1990), in IAU Symp. No. 141, Inertial Coordinate System on the Sky, held in Leningrad, USSR, 17-21 October 1989, eds. J.H. Lieske, V.A. Abalakin, p. 371

Schwan, H. (1991), in IAU Coll. No. 100, Fundamentals of Astrometry, held in Belgrade, Yugoslavia, 8-11 September 1987, eds. H.K. Eichhorn, C.A. Murray, Astrophys. Space Sci. No. 177 , p. 23

Smith, C. (1980), in Mitteilungen der Astron. Gesellschaft, No. 48, Astrometrie und Dynamische Astronomie, Hamburg, p. 95

Smith, C., Corbin, C., Hughes, J., Jackson, E., Khrutskaya, A., Polozhentsev, A., Polozhentsev, D., Yagudin, L., Zverev, M. (1990), in IAU Symp. No. 141, Inertial Coordinate System on the $S k y$, held in Leningrad, USSR, 17-21 Oct. 1989, eds. J.H. Lieske, V.A. Abalakin, p. 457

Williams, J,G., Newhall, X.X., Dickey, J.,O. (1991), Astron. Astrophys., Vol. 241, L9

Wahl, E. (1937), Astron. Nachr., Vol. 263, 249

Yoshizawa, M., Suzuki, S., Fukaya, R., (1987), Ann. Tokyo Astron. Obs., 2nd Series, Vol. 21, p. 399

Yoshizawa, M., Suzuki, S. (1989), Publ. Natl. Astron. Obs. Japan, Vol. 1, p. 127

Yoshizawa, M., Suzuki, S. (1991), Publ. Natl. Astron. Obs. Japan, Vol. 2, p. 103

Yoshizawa, M., Suzuki, S., Sôma, M. (1992), Publ. Natl. Astron. Obs. Japan, Vol. 2, p. 475 